\title{
CONDUCTAS DE APRENDIZAJE EN NIÑOS CON TRASTORNO DEL ESPECTRO AUTISTA CON Y SIN COMORBILIDAD CON DÉFICIT DE ATENCIÓN E HIPERACTIVIDAD
}

\author{
Carmen Berenguer \\ Psicología Evolutiva y de la Educación. Universidad de Valencia \\ carmen.berenguer@uv.es \\ Belén Roselló \\ Psicología Evolutiva y de la Educación. Universidad de Valencia \\ Inmaculada Baixauli \\ Universidad Católica de Valencia
}

Fecha de Recepción: 4 Febrero 2018

Fecha de Admisión: 10 Abril 2018

\section{RESUMEN}

Introducción. La literatura ha explorado los problemas en el aprendizaje que presentan los niños con trastorno del espectro autista (TEA), pero apenas se ha analizado el impacto de la comorbilidad con déficit de atención e hiperactividad (TDAH) sobre el aprendizaje en niños con TEA. Objetivos. 1) Analizar las diferencias en la conducta de aprendizaje de niños con TEA con y sin comorbilidad con TDAH y niños con desarrollo típico (TD); 2) Investigar la asociación entre diferentes dominios de conductas de aprendizaje y problemas conductuales y emocionales, en niños con TEA y niños con TEA+TDAH. Método. Participaron 87 niños (30 con diagnóstico de TEA de alto funcionamiento, 22 con TEA+TDAH y 36 con TD entre 7 y 11 años igualados en edad, capacidad cognitiva y vocabulario. Los profesores informaron sobre la escala de conductas de aprendizaje (LBS), y los padres cumplimentaron el cuestionario de Fortalezas y Dificultades (SDQ). Resultados. Los resultados mostraron diferencias significativas entre los niños con TEA con y sin comorbilidad en todos los dominios del LBS. A su vez las correlaciones revelaron que a menor flexibilidad en la búsqueda de estrategias mayores problemas emocionales en el TEA y mayores problemas conductuales en el TEA+TDAH. Conclusiones. Los hallazgos ponen de manifiesto el impacto negativo de los síntomas de TDAH en todos los prerrequisitos de aprendizaje analizados. Así mismo los datos mostraron la implicación de los problemas emocionales y comportamentales en la capacidad de cambio a la hora de planificar estrategias de aprendizaje en niños con autismo de alto funcionamiento.

Palabras clave: aprendizaje; comorbilidad; LBS; problemas conductuales; TEA; TDAH

\section{ABSTRACT}

Learning behaviors in children with autism spectrum disorder with and without comorbidity with attention deficit hyperactivity disorder. 


\section{CONDUCTAS DE APRENDIZAJE EN NIÑOS CON TRASTORNO DEL ESPECTRO AUTISTA CON Y SIN COMORBILIDAD CON DÉFICIT DE ATENCIÓN E HIPERACTIVIDAD}

Introduction. The literature has explored the learning problems presented by children with autism spectrum disorder (ASD), but the impact of comorbidity with attention deficit hyperactivity disorder (ADHD) on learning in children with ASD has barely been analyzed. Goals. 1) To analyze the differences in the learning behavior of children with ASD with and without comorbidity with ADHD and children with typical development (TD); 2) Investigate the association between different domains of learning behaviors and behavioral and emotional problems in children with ASD and children with ASD + ADHD. Method. 87 children participated (30 with a diagnosis of high functioning ASD, 22 with ASD + ADHD and 36 with TD between 7 and 11 years matched in age, cognitive ability and vocabulary.) Teachers reported on the scale of learning behaviors (LBS), and the parents completed the Strengths and Difficulties questionnaire (SDQ) Results The results showed significant differences between children with ASD with and without comorbidity in all LBS domains, and correlations revealed that less flexibility in the search of strategies for greater emotional problems in ASD and greater behavioral problems in ASD + ADHD Conclusions The findings highlight the negative impact of ADHD symptoms on all the learning prerequisites analyzed. emotional and behavioral problems in the ability to change when planning learning strategies in children with autism high performance.

Keywords: learning; ASD; ADHD; comorbidity; LBS; behavioral problems

\section{ANTECEDENTES}

Los déficits en la comunicación e interacción social y la existencia de patrones de conducta, intereses 0 actividades restringidos y repetitivos son característicos de los niños con trastorno del espectro del autismo (TEA). El TEA es un trastorno del neurodesarrollo con una prevalencia que actualmente se estima en torno al 2\% en población escolar (Blumberg et al., 2013). Este incremento de prevalencia se debe en parte al mayor reconocimiento de los síntomas de TEA en niños con capacidad cognitiva dentro de los límites de la normalidad (Joshi et al., 2017). Además, el TEA presenta una elevada comorbilidad clínica. En concreto entre el 37-85\% de niños con TEA presentan síntomas comórbidos de trastorno por déficit de atención con hiperactividad TDAH (Leitner, 2014). Desde que el DSM-5 (APA, 2013) permitió por primera vez el diagnóstico conjunto de TEA y déficit de atención con hiperactividad (TEA+TDAH), se ha incrementado el interés en conocer los aspectos característicos de este nuevo grupo (Antshel, Zhang-James, Wagner, Ledesma, y Faraone, 2016).

Los síntomas nucleares del TEA y sus características asociadas pueden tener importantes repercusiones en la conducta de aprendizaje. La literatura pone de manifiesto que los niños con TEA de alto funcionamiento presentan un elevado riesgo de dificultades académicas y como consecuencia pueden estar menos preparados que sus pares con desarrollo típico (DT) (Griswold, Barnhill, Myles, Hagiwara, \& Simpson, 2002). A pesar de la enorme variabilidad que presenta el TEA, las estimaciones sugieren que un alto porcentaje de los niños con TEA de alto funcionamiento se desempeñan peor de lo esperado en al menos un área académica y tienen 5.5 veces más probabilidades de mostrar dificultades de aprendizaje (Estes, Rivera, Bryan, Cali, \& Dawson, 2011; Jones et al., 2009).

Las causas de las dificultades académicas dependen de muchos factores. La competencia académica es un constructo multidimensional compuesto de habilidades académicas y prerrequisitos académicos, es decir conductas de aprendizaje que pueden facilitar o interferir el desarrollo escolar (DiPerna \& Elliott, 2002). Estas conductas de aprendizaje están relacionadas con la motivación, la actitud hacia el aprendizaje, la persistencia en las tareas y la búsqueda de estrategias (McDermott, Rikoon, \& Fantuzzo, 2016), Así como también en las relaciones con los compañeros, o los problemas de conducta y emocionales (Mendelson, Gates, \& Lerner, 2016).

La literatura ha evidenciado que los niños con TEA presentan una actitud más negativa hacia el aprendizaje, tienen menos relaciones de amistad y una menor calidad en las relaciones con sus compañeros dentro del entorno escolar (Chien, Tu, \& Gau, 2017). Además, algunos estudios ponen de 
relieve que los síntomas de autismo relacionados con los déficits sociales incrementan el riesgo de mayor desajuste académico, que se manifiesta por una actitud negativa hacia las tareas escolares, 0 las asignaturas donde es importante la capacidad para cambiar las estrategias de aprendizaje, especialmente en la resolución de problemas (McDermott et al., 2016).

Por su parte, la presencia comórbida de TDAH (TEA+TDAH), parece que produce mayores dificultades en el funcionamiento de áreas relacionadas con las conductas de aprendizaje (Sikora, Vora, Coury, \& Rosenberg, 2012), socialización o problemas emocionales y de comportamiento (Rao \& Landa, 2014). En concreto, desde una aproximación dimensional, diversos estudios sugieren que son los síntomas de inatención más que los de hiperactividad, impulsividad los que producen más deterioro en el funcionamiento social y en el éxito académico (Chiang \& Gau, 2015). Aunque otros estudios como el de Mattila et al. (2010) no han encontrado mayor deterioro en general en el grupo TEA+TDAH.

En resumen, los niños con TEA de alto funcionamiento presentan un elevado riesgo de presentar problemas en su conducta de aprendizaje y la presencia de síntomas comórbidos de TDAH puede producir mayores dificultades. Pocos estudios han relacionado las conductas de aprendizaje con los problemas de conducta y emocionales que presentan los niños con TEA y ninguno hasta el momento ha abordado este tema en el grupo comórbido TEA+TDAH. De este modo que los objetivos del presente estudio son:

1) Analizar las diferencias en la conducta de aprendizaje de niños con TEA con y sin comorbilidad con TDAH y niños con desarrollo típico (TD).

2) Investigar la asociación entre diferentes dominios de conductas de aprendizaje y problemas conductuales y emocionales, en niños con TEA y niños con TEA+TDAH.

En base a la literatura anterior, se espera que los síntomas de TDAH producirán un mayor déficit en las conductas de aprendizaje de los niños con TEA. Así mismo se hipotetiza que las deficiencias en las conductas de aprendizaje estarán relacionadas con los problemas de conducta y emocionales de los niños con TEA, especialmente cuando coexistan ambos trastornos (TEA+TDAH).

\section{MÉTODO}

\section{Participantes}

En este trabajo participaron 89 niños con edades comprendidas entre los 7 y 11 años, escolarizados en centros de enseñanza públicos y concertados. Todos los participantes tenían una capacidad intelectual (CI) dentro de los límites de la normalidad, medida mediante el K-BIT (Kaufman \& Kaufman, 2000) y estaban distribuidos en tres grupos; un grupo de 30 niños con Trastorno del espectro autista de alto funcionamiento (TEA), 22 niños con TEA y trastorno por déficit de atención con hiperactividad comórbido (TEA+TDAH) y 37 niños con desarrollo típico (TD), igualados en edad y $\mathrm{Cl}$. El $79.7 \%$ de los participantes eran varones, y un $20.3 \%$ mujeres. El lenguaje fue evaluado mediante la subprueba del vocabulario del WISC-IV (Wechsler, 2003) (ver Tabla 1). 


\section{CONDUCTAS DE APRENDIZAJE EN NIÑOS CON TRASTORNO DEL ESPECTRO AUTISTA CON Y SIN COMORBILIDAD CON DÉFICIT DE ATENCIÓN E HIPERACTIVIDAD}

Tabla 1. Datos socio-demográficos

\begin{tabular}{lrrrrrl}
\hline & TD $(\mathrm{n}=37)$ & TEA(n=30) & TEA+(n=22) & & & \\
& Media (dt) & Media (dt) & Media (dt) & $F_{2,86}$ & \multicolumn{1}{c}{$\chi^{2}$} & \multicolumn{1}{l}{$p$} \\
\hline Edad (años) & $8.5(1.2)$ & $8.4(1.3)$ & $8.8(1.3)$ & .78 & - & .460 \\
CI & $102.1(8.9)$ & $100.4(12.4)$ & $102.9(13.0)$ & .35 & - & .705 \\
Vocabulario & $12.9(2.7)$ & $11.5(3.3)$ & $11.4(3.4)$ & 2.16 & - & .121 \\
Nivel educativo p & $3.5(.9)$ & $3.4(1.0)$ & $3.1(1.2)$ & .78 & - & .459 \\
Genero (\% H) & $62.1 \%$ & $90.0 \%$ & $95.4 \%$ & - & 12.41 & $.002^{*}$ \\
Medicación (\% sí) & $0.0 \%$ & $26.6 \%$ & $40.9 \%$ & - & 16.61 & $.000^{*}$ \\
\hline$* p<.05$ & & & & & &
\end{tabular}

Nivel educativo $\mathrm{p}$ (nivel educativo padres) se tomó el nivel más alto del padre o de la madre $(0=$

Educación primaria, 1= Educación secundaria, 2= FPI, 3=FPII, Bachiller, 4= Universitario)

Grupo TEA y grupo TEA+TDAH. Los participantes con TEA con y sin comorbilidad con TDAH habían recibido un diagnóstico clínico en los servicios de Psiquiatría y Neuropediatría de hospitales y centros de salud de la Comunidad Valenciana. Con el objetivo de confirmar el diagnóstico de TEA se aplicaron los puntos de corte estrictos que son recomendados en el Cuestionario de Comunicación Social (SCQ; Rutter, Bailey, \& Lord, 2003), y en la entrevista diagnóstica de autismo revisada (ADI-R; Rutter, Le Couteur \& Lord, 2003). Las medidas diagnósticas fueron administradas por una psicóloga acreditada en su aplicación, que formaba parte del equipo de investigación. El $38.46 \%$ de los niños con TEA con y sin comorbilidad estaban escolarizados en Aulas específicas de Comunicación y Lenguaje y el $61.54 \%$ estaban escolarizados en aulas ordinarias y asistían cada día a aulas de apoyo terapéutico a tiempo parcial en el mismo centro donde estaban escolarizados. Además, con el objetivo de confirmar los síntomas comórbidos de TDAH, los padres y profesores cumplimentaron los 18 criterios para el TDAH del DSM-5 (APA, 2013).

Grupo Control. Los niños con desarrollo típico (DT) estaban escolarizados en aulas ordinarias de los centros escolares a los que asistían los niños diagnosticados de TEA. No tenían historia de psicopatología ni de remisión a unidades de salud mental infantil (USMI) según la información que constaba en los registros escolares y tampoco cumplían criterios del DSM-5 de TEA en el screening que se llevó a cabo antes de comenzar la evaluación. Los criterios de exclusión para los niños que participaron en esta investigación fueron valorados a través de una extensa anamnesis previa realizada con las familias. Comprendían enfermedades neurológicas o genéticas, lesión cerebral, déficits sensoriales visuales, auditivos o motores y un cociente intelectual inferior a 80. La administración de psicofármacos solo fue un criterio de exclusión para los niños del grupo con desarrollo típico.

\section{Instrumentos}

Escala de conductas de aprendizaje (Learning Behaviors Scale; LBS; McDermott, Green, Francis, \& Stott, 2001). Es un cuestionario estandarizado de 29 ítems dirigido a profesores. Su objetivo es evaluar los comportamientos relacionados con el aprendizaje efectivo del estudiante durante los últimos 2 meses. El LBS ofrece 4 dimensiones y una puntuación total; Motivación, Actitud hacia el aprendizaje, Persistencia en la tarea, Estrategias de aprendizaje/Flexibilidad. Puntuaciones altas indican una buena conducta de aprendizaje. Las puntuaciones directas se convierten en puntuaciones T normalizadas (media $=50$, desviación típica $=10$ ), basado en la estandarización de una muestra representativa de 1.500 estudiantes con edades comprendidas entre 5 y 17 años. Los coeficientes de consistencia interna son altos para la puntuación total $(.89, .92)$ y para las subescalas $(.70, .87)$ (McDermott, Rikoon, Waterman, \& Fantuzzo, 2011).

Cuestionario de Capacidades y Dificultades (Strengths and Difficulties Questionnaire; SDQ; 
Goodman, 1997). Se trata de un cuestionario de 25 ítems dirigido a niños entre 4 y 16 años. Los ítems están divididos en 5 subescalas: Síntomas emocionales, Problemas de conducta, Hiperactividad, Relaciones con los compañeros y Conductas prosociales. Además, cuenta con una puntuación total de dificultad creada mediante la suma de todas las subescalas excepto la de Conducta prosocial. Existen tres versiones del cuestionario: padres, profesores y autoinforme, en esta investigación el SDQ fue cumplimentado por los padres. Los ítems se puntúan en una escala tipo Likert que va de 1 (no es cierto) a 3 (completamente cierto). En todas las escalas una puntuación superior implica mayor problematicidad a excepción de la escala Conducta prosocial, donde una puntuación mayor es más positiva que una menor. El SDQ fue cumplimentado por los padres. EL SDQ cuenta con propiedades estadísticas y psicométricas adecuadas en la población española (Rodríguez-Hernández et al., 2012) y en muestras con TEA (Findon et al., 2016).

\section{Procedimiento}

Este estudio contó con la aprobación del Comité Ético para la investigación con humanos de la Universidad de Valencia (Declaration of Helsinki en la Convention of the European Council, 1964). Así mismo se obtuvo la autorización de la Consejería de educación de la Generalitat Valenciana, para acceder a los colegios y localizar a los niños que habían recibido previamente un diagnóstico de TEA con y sin comorbilidad con TDAH por profesionales especializados en los servicios de salud pública infantil. Se obtuvo el consentimiento oral y escrito de todos los padres de los niños incluidos en el estudio, una vez que se les informó de los objetivos de la investigación. La evaluación se llevó a cabo en los mismos colegios donde los niños estaban escolarizados, y se realizó en aulas preparadas especialmente para garantizar las condiciones adecuadas durante la evaluación. Para la realización del presente estudio padres y profesores cumplimentaron unos cuestionarios administrados por una psicóloga que formaba parte del equipo de investigación.

\section{Análisis de datos}

Los análisis estadísticos se realizaron con el software Statistical Package for the Social Science (SPSS), versión 22.00 (SPSS Inc., Chicago, IL USA). Para analizar las diferencias entre los grupos TEA, TEA+TDAH y desarrollo típico en los diferentes dominios del LBS se realizó un análisis multivariado de la varianza (MANOVA). Las diferencias en el total de la prueba se comprobaron mediante análisis univariados de la varianza (ANOVAs) adicionales. En ambos casos, se estableció el nivel de significación en .05 y se calculó el valor de 2 para comprobar la fuerza de asociación. Posteriormente se realizaron dos correlaciones de Pearson (una con el grupo TEA y la otra con el grupo TEA+TDAH) utilizando las puntuaciones t de los dominios del LBS (Motivación, actitud hacia el aprendizaje, persistencia, estrategias y el total del LBS) y las puntuaciones directas de las subescalas del SDQ con el fin de explorar las asociaciones entre las conductas de aprendizaje y los problemas comportamentales, emocionales junto con la conducta prosocial en ambos grupos clínicos.

\section{RESULTADOS}

\section{Perfil de conductas de aprendizaje de niños con TEA, TEA+TDAH y desarrollo típico}

El MANOVA realizado entre los grupos TEA, TEA+TDAH y TD con las puntuaciones $t$ del LBS resultó estadísticamente significativo (Lambda de Wilks $=.42 ; \mathrm{F}_{10,164}=8.84 ; p<.01 ; \eta^{2} p=.35$ ). En concreto, se encontraron diferencias estadísticamente significativas entre las puntuaciones diferenciales de los diferentes grupos en Motivación ( $\left.F_{2.86}=22.49 ; p<.01 ; \eta^{2} p=.343\right)$, Actitud $\left(F_{2,86}=28.21 ; p\right.$ $\left.<.01 ; \eta^{2} p=.396\right)$, Persistencia $\left(F_{2,86}=33.74 ; p<.01 ; \eta^{2} p=.440\right)$, Estrategias $\left(F_{2,86}=40.73 ; p<\right.$ $\left..01 ; \eta^{2} p=.486\right)$, y la puntuación total del LBS $\left(F_{2,86}=31.68 ; p<.01 ; \eta^{2} p=.424\right)$. Por su parte, los 


\section{CONDUCTAS DE APRENDIZAJE EN NIÑOS CON TRASTORNO DEL ESPECTRO AUTISTA CON Y SIN COMORBILIDAD CON DÉFICIT DE ATENCIÓN E HIPERACTIVIDAD}

análisis post hoc de comparaciones múltiples realizados utilizando la corrección de Bonferroni revelaron la existencia de diferencias significativas entre el grupo con TD y los otros dos grupos clínicos en todas las subescalas y en la escala total de conductas de aprendizaje. Del mismo modo, se evidenciaron diferencias significativas entre el grupo con TEA puro y el grupo con TEA+TDAH en todas las subescalas y en la escala total del LBS, donde el grupo con TEA+TDAH presentó las puntuaciones medias más bajas (ver Tabla 2).

Tabla 2. Diferencias entre los grupos de niños TD, TEA, y TEA+TDAH en las subescalas del LBS (Anovas)

\begin{tabular}{|c|c|c|c|c|c|c|}
\hline & $\begin{array}{c}\text { TD } \\
(n=37)\end{array}$ & $\begin{array}{c}\text { TEA } \\
(\mathrm{n}=30)\end{array}$ & $\begin{array}{c}\text { TEA+TDAH } \\
(\mathrm{n}=22)\end{array}$ & $F_{(2,86)}$ & $\eta_{\mathrm{P}}^{2}$ & $\begin{array}{c}\text { Diferencias de } \\
\text { grupo }\end{array}$ \\
\hline$L B S$ & $\mathrm{M}(\mathrm{DT})$ & $\mathrm{M}$ (DT) & $\mathrm{M}$ (DT) & & & \\
\hline Motivación & $53.1(9.7)$ & $42.7(10.7)$ & $35.2(10.3)$ & $22.49 *$ & .343 & $\begin{array}{l}\text { TEA+TDAH } \\
<\text { TEA, TD }\end{array}$ \\
\hline Actitud & $50.5(7.8)$ & $42.4(6.3)$ & $36.4(7.2)$ & $28.21 *$ & .396 & $\begin{array}{l}\text { TEA+TDAH } \\
<\text { TEA, TD }\end{array}$ \\
\hline Persistencia & $52.4(8.4)$ & $43.8(6.6)$ & $36.8(5.5)$ & $33.74 *$ & .440 & $\begin{array}{l}\text { TEA+TDAH } \\
<\text { TEA, TD }\end{array}$ \\
\hline Estrategia & $50.4(8.1)$ & $35.2(11.4)$ & $22.1(16.8)$ & $40.73 *$ & .486 & $\begin{array}{l}\text { TEA+TDAH } \\
<\text { TEA, TD }\end{array}$ \\
\hline LBS Total & $52.1(9,1)$ & $40.6(9.8)$ & $28.7(14.8)$ & $31.68 *$ & .424 & $\begin{array}{l}\text { TEA+TDAH } \\
<\text { TEA, TD }\end{array}$ \\
\hline
\end{tabular}

$* p<.001$

LBS (learning behaviour scale), TD (desarrollo típico), M (media), DT (desviación típica)

\section{Relación entre las conductas de aprendizaje y los problemas comportamentales y emocionales} de niños con TEA y niños con TEA+TDAH

Para analizar la asociación entre las conductas de aprendizaje y los problemas comportamentales, emocionales, así como la socialización de niños con TEA con y sin comorbilidad con TDAH, se realizaron dos correlaciones de Pearson.

En el grupo de niños con TEA puro, se observaron correlaciones positivas significativas entre la puntuación $t$ de estrategias de aprendizaje y los problemas emocionales $(r=.51 ; p<.01)$ y la subescala de total de dificultades del SDQ $(r=.37 ; p=.04)$. Del mismo modo se observaron correlaciones positivas signficativas entre la subescala de problemas emocionales y el total de conductas de aprendizaje $(r=.41 ; p=.02)$.

Por su parte, en el grupo comórbido de niños con TEA+TDAH se observaron correlaciones positivas significativas entre la subescala de estrategias de aprendizaje y los problemas comportamentales del SDQ $(r=.53 ; p=.01)$. 
Tabla 3. Correlaciones entre las subescalas del LBS-profesor y del SDQ-padres en niños con TEA y TEA+TDAH

\begin{tabular}{|c|c|c|c|c|c|c|c|c|c|c|c|}
\hline $\begin{array}{l}\text { TEA+TDAH } \\
\text { TEA }\end{array}$ & 1 & 2 & 3 & 4 & 5 & 6 & 7 & 8 & 9 & 10 & 11 \\
\hline 1.Motivación & - & $.65^{* *}$ & .29 & .18 & $.75 * *$ & -.31 & -.2 & -.33 & -.09 & .18 & -.39 \\
\hline 2.Actitud & $.60 * *$ & - & $.64 * *$ & $.54 * *$ & $.81 * *$ & -.12 & -.18 & -.16 & -.12 & $-0,11$ & -.24 \\
\hline 3.Persistencia & $.52 * *$ & $.64 * *$ & - & $.54 * *$ & $.56^{* *}$ & .30 & -.25 & .01 & .04 & -.18 & .05 \\
\hline 4.Estrategia & .27 & $.39 *$ & $.38^{*}$ & - & $.58 * *$ & .23 & $.53 *$ & -.31 & -.15 & .01 & -.26 \\
\hline 5.LBS Total & $.89 * *$ & $.71^{* *}$ & $.60 * *$ & $.46^{* *}$ & - & -.07 & -.30 & -.39 & -.08 & -.01 & -.32 \\
\hline 6.P. Emocional & .33 & .09 & .16 & $.51^{* *}$ & $.41^{*}$ & - & -.07 & .25 & $.46^{*}$ & -.28 & $.72 * *$ \\
\hline 7.P. Comporta & .33 & .11 & .10 & -.03 & .20 & $.45^{*}$ & - & $.45^{*}$ & -.03 & -.49 & $.49^{*}$ \\
\hline 8.P. Hiperactivi & .29 & .04 & .11 & .27 & .27 & $.40 *$ & .30 & - & -.02 & -.35 & $.65^{* *}$ \\
\hline 9.P. Iguales & .04 & -.03 & .09 & .26 & .07 & .34 & .21 & .27 & - & -.14 & $.57 * *$ \\
\hline 10.Prosocial & -.26 & -.07 & -.09 & -.07 & -.07 & $-.39 *$ & $-.43 *$ & -.33 & $-.37 *$ & - & -.51 \\
\hline 11.T. Dificult & .35 & .06 & .06 & $.37 *$ & .33 & $.75 * *$ & $.64^{* *}$ & $.75^{* *}$ & $.66^{* *}$ & $\stackrel{-}{53^{* *}}$ & - \\
\hline
\end{tabular}

LBS (learning behaviour scale), P. (problemas), Comporta (comportamentales), Hiperactivi (hiperactividad), T. Dificult (total de dificultades del SDQ)

\section{CONCLUSIONES}

El objetivo principal de este trabajo fue explorar las conductas de aprendizaje relacionadas con la motivación, la actitud hacia las tareas escolares, la persistencia en la tarea y la capacidad de generar diferentes estrategias en el aprendizaje, en niños con autismo puro y niños con autismo con síntomas comórbidos de hiperactividad e impulsividad (TEA+TDAH). Para ello se abordaron dos objetivos: El primer objetivo del estudio fue analizar las diferencias en la conducta de aprendizaje de niños con TEA, TEA+TDAH y desarrollo típico. Los resultados mostraron tal y como se esperaba que los niños con desarrollo típico presentaban mejores conductas de aprendizaje, valoradas por los profesores, con respecto a los niños con autismo con y sin comorbilidad con TDAH. Estos hallazgos se sitúan en la línea de los encontrados en otros estudios que han comparado niños con desarrollo típico y niños con TEA de alto funcionamiento (Estes et al., 2011; Griswold et al., 2002). Por otra parte, los resultados revelaron más dificultades en la conducta de aprendizaje valorada por los profesores, en el grupo con TEA+TDAH cuando se comparó con el grupo TEA puro. Hallazgos en parte parecidos a un reciente estudio que utilizó un cuestionario cumplimentado por padres para valorar el ajuste social en diferentes áreas de funcionamiento (Chiang et al., 2017). Los resultados indicaron la existencia de más déficits en el funcionamiento escolar en los niños con TEA+TDAH, en relación a los niños con TEA puro. En concreto, Ios estudiantes con TEA+TDAH tuvieron una peor actitud hacia los deberes escolares, menos interacciones con sus profesores y más problemas de comportamiento en el colegio. Estos hallazgos ponen de manifiesto el impacto negativo que tienen los síntomas comórbidos de TDAH en niños y adolescentes con TEA en dominios relacionados con el funcionamiento escolar (Sikora et al., 2012). Los problemas de autorregulación emocional y una mayor aversión a la demora podrían explicar en parte, la mayor afectación de este grupo comórbido en la actitud hacia el aprendizaje y los problemas de comportamiento (Chiang et al., 2017). 


\section{CONDUCTAS DE APRENDIZAJE EN NIÑOS CON TRASTORNO DEL ESPECTRO AUTISTA CON Y SIN COMORBILIDAD CON DÉFICIT DE ATENCIÓN E HIPERACTIVIDAD}

El segundo objetivo se propuso investigar la asociación entre diferentes dominios de conductas de aprendizaje y problemas sociales, conductuales y emocionales, en niños con TEA y niños con TEA+TDAH. Los resultados evidenciaron un perfil similiar entre el grupo TEA y el grupo comórbido. De manera que se encontraron correlaciones estadísticamente significativas solamente entre algunos de los dominios del SDQ, específicamente aquellos relacionados con los problemas emocionales y de conducta y la capacidad de cambio a la hora de generar estrategias de aprendizaje, tanto en el grupo TEA como en el grupo TEA+TDAH. En concreto, la subescala de estrategias de aprendizaje presentó una asociación significativa con los problemas emocionales en el grupo TEA puro y con los problemas comportamentales en el grupo TEA+TDAH. La subescala de estrategias está relacionada con el dominio ejecutivo de flexibilidad cognitiva, es decir, la capacidad de cambiar de un pensamiento a otro, de una tarea a otra y usar la flexibilidad para resolver un problema en diversos contextos (Lawson et al., 2015). Precisamente uno de los síntomas característicos del autismo son las dificultades que presentan en la flexibilidad cognitiva. En esta línea, algunos trabajos han mostrado la relación entre las dificultades en la capacidad para generar estrategias y los problemas emocionales y de conducta en niños con TEA (Chien et al., 2017).

Otros estudios por el contrario, sí han hallado una asociación entre aspectos relacionados con la motivación hacia el aprendizaje y una mejora en los problemas de conducta en niños con TEA (Koegel, Singh, \& Koegel, 2010). Diferencias en el tipo de medida de evaluación utilizada y las características de los participantes, ya que en el mencionado estudio, participaron niños entre 4 y 7 años, podrían explicar en parte, estas divergencias, en cuanto a los resultados obtenidos.

Por lo tanto, el presente trabajo evidencia el impacto negativo de los síntomas de TDAH en todos los prerrequisitos de aprendizaje analizados. Así como, la implicación de los problemas emocionales y comportamentales en la capacidad de cambio a la hora de planificar estrategias de aprendizaje en niños con autismo de alto funcionamiento.

Este estudio presenta algunas limitaciones, como el reducido tamaño de la muestra y el criterio de incluir solamente niños con TEA de alto funcionamiento. Por lo que no podrían generalizarse los resultados a niños con TEA que tengan una capacidad cognitiva menor. Otra de las limitaciones se refiere a la utilización de diferentes informantes para valorar las conductas de aprendizaje, por un lado y los problemas relacionados con aspectos sociales y comportamentales, por otro. Por lo tanto, sería necesario complementar la información de las conductas de aprendizaje, con la percepción de los padres y a su vez valorar los problemas comportamentales y sociales desde el punto de vista de los profesores.

Los hallazgos obtenidos tienen implicaciones para la evaluación e intervención psicopedagógica de niños con TEA de alto funcionamiento. La detección de los síntomas comórbidos de TDAH en los niños con TEA es un aspecto clave por las importantes repercusiones en el diseño de programas de intervención adecuados. Además, las conductas de aprendizaje están más afectadas en niños con autismo y síntomas clínicos de TDAH, por lo que es necesario abordar los síntomas de inatención especialmente, para mejorar el funcionamiento escolar de los niños con TEA.

\section{REFERENCIAS}

American Psychiatric Association. (2013). Diagnostic and statistical manual of mental disorders (5th ed.). Arlington, VA: American Psychiatric Publishing.

Antshel, K. M., Zhang-James, Y., Wagner, K. E., Ledesma, A., \& Faraone, S. V. (2016). An update on the comorbidity of ADHD and ASD: A focus on clinical management. Expert Review of Neurotherapeutics, 16, 279-293.

Blumberg, S. J., Bramlett, M. D., Kogan, M. D., Schieve, L. A., Jones, J. R., \& Lu, M. C. (2013). Changes in prevalence of parent-reported autism spectrum disorder in school-aged U.S. children: 
2007 to 2011-2012 (National Health Statistics Report No. 65). Retrieved from http://www.cdc.gov/nchs

Chiang, H. L., Kao, W. C., Chou, M. C., Chou, W. J., Chiu, Y. N., Wu, Y. Y., \& Gau, S. S. F. (2017). School dysfunction in youth with autistic spectrum disorder in Taiwan: The effect of subtype and ADHD. Autism Research. https://doi.org/10.1002/aur.1923

Chien, Y. L., Tu, E. N., \& Gau, S. S. F. (2017). School Functions in Unaffected Siblings of Youths with Autism Spectrum Disorders. Journal of Autism and Developmental Disorders, 47, 3059-3071.

DiPerna, J. C., \& Elliott, S. N. (2002). Promoting academic enablers to improve student achievement: an introduction to the miniseries. Sch. Psychol. Rev. 31, 293-297.

Estes, A., Rivera, V., Bryan, M., Cali, P., \& Dawson, G. (2011). Discrepancies between academic achievement and intellectual ability in higher-functioning school-aged children with autism spectrum disorder. Journal of Autism and Developmental Disorders, 41, 1044-1052.

Findon, J., Cadman, T., Stewart, C. S., Woodhouse, E., Eklund, H., Hayward, H., ... \& Murphy, D. (2016). Screening for co occurring conditions in adults with autism spectrum disorder using the strengths and difficulties questionnaire: A pilot study. Autism Research, 9, 1353-1363.

Goodman, R. (1997). The Strengths and Difficulties Questionnaire: A research note. Journal of Child Psychology and Psychiatry, 38, 581-586.

Griswold, D. E., Barnhill, G. P., Myles, B. S., Hagiwara, T., \& Simpson, R. L. (2002). Asperger syndrome and academic achievement. Focus on Autism and Other Developmental Disabilities, 17, 94-102.

Jones, C.R., Happe, F., Golden, H., Marsden, A.J., Tregay, J., Simonoff, E., . . Charman, T. (2009). Reading and arithmetic in adolescents with autism spectrum disorders: Peaks and dips in attainment. Neuropsychology, 23, 718-728.

Joshi, G., Faraone, S. V., Wozniak, J., Tarko, L., Fried, R., Galdo, M., ... \& Biederman, J. (2017). Symptom profile of ADHD in youth with high-functioning autism spectrum disorder: a comparative study in psychiatrically referred populations. Journal of Attention Disorders, 21(10), 846-855.

Kaufman, A. S., \& Kaufman, N. I. (2000). K-BIT, Test breve de inteligencia de Kauffman [Kaufman brief intelligence test]. Madrid: Pearson.

Koegel, L. K., Singh, A. K., \& Koegel, R. L. (2010). Improving motivation for academics in children with autism. Journal of Autism and Developmental Disorders, 40, 1057-1066.

Lai, M.C., Lombardo, M.V., \& Baron-Cohen, S. (2014). Autism. Lancet, 383, 896-910.

Lawson, R. A., Papadakis, A. A., Higginson, C. I., Barnett, J. E., Wills, M. C., Strang, J. F., ... \& Kenworthy, L. (2015). Everyday executive function impairments predict comorbid psychopathology in autism spectrum and attention deficit hyperactivity disorders. Neuropsychology, 29, 445-453.

Leitner Y. (2014). The co-occurrence of autism and attention deficit hyperactivity disorder in children - what do we know? Frontiers in Human Neuroscience 8, 268.

Mattila, M.L., Hurtig, T., Haapsamo, H., Jussila, K., Kuusikko-Gauffin, S., Kielinen, M., . . Moilanen, I. (2010). Comorbid psychiatric disorders associated with Asperger syndrome/high-functioning autism: a community- and clinic-based study. Journal of Autism and Developmental Disorders, 40, 1080-1093.

McDermott, P. A., Green, L. F., Francis, J. M., \& Stott, D. H. (2001). Learning Behaviors Scale. Philadelphia, PA: Edumetric and Clinical Science

McDermott, P. A., Rikoon, S. H., \& Fantuzzo, J. W. (2016). Transition and protective agency of early childhood learning behaviors as portents of later school attendance and adjustment. Journal of School Psychology, 54, 59-75.

McDermott, P. A., Rikoon, S. H., Waterman, C., \& Fantuzzo, J. W. (2011). The preschool learning 
behaviors scale: dimensionality and external validity in head start. Journal of School Psychology, $41,66-81$.

Mendelson, J.L., Gates, J.A., \& Lerner, M.D. (2016). Friendship in school-age boys with autism spectrum disorders: A metaanalytic summary and developmental, process-based model. Psychological Bulletin, 142, 601-622.

Rao, P.A., \& Landa, R.J. (2014). Association between severity of behavioral phenotype and comorbid attention deficit hyperactivity disorder symptoms in children with autism spectrum disorders. Autism, 18, 272-280.

Rodríguez-Hernández, P. J., Betancort, M., Ramírez-Santana, G. M., García, R., Sanz-Álvarez, E.J., \& De las Cuevas-Castresana, C. (2012). Psychometric properties of the parent and teacher versions of the strength and difficulties questionnaire (SDQ) in a spanish sample. International Journal of Clinical and Health Psychology, 12, 265-279.

Rutter, M., Bailey, A., \& Lord, C. (2003). Social communication questionnaire. Los Angeles: Western Psychological Services.

Rutter, M., Le Couteur, A., \& Lord, C. (2003). ADI-R. Autism diagnostic interview revised. Manual. Los Angeles: Western Psychological Services

Sikora, D.M., Vora, P., Coury, D.L., \& Rosenberg, D. (2012). Attention-deficit/hyperactivity disorder symptoms, adaptive functioning, and quality of life in children with autism spectrum disorder. Pediatrics, 130, S91-S97.

Wechsler, D. (2003). Wechsler intelligence scale for children, fourth edition (WISC-IV). San Antonio, TX: The Psychological Corporation. 